\title{
Predictors of Progression of Coronary Atherosclerosis after Percutaneous Coronary Intervention
}

\author{
Violeta Dinesch1, Mihail Dinesch², Ileana Voichita Sirbu³, Cosmin Macarie ${ }^{3}$, Bogdan Vasile Halatiu, \\ Mircea Buruian 5 \\ 1. University of Medicine and Pharmacy Tirgu Mures, Romania \\ 2. Department of Cardiology, Institute for Cardiovascular Disease and Heart Transplant, Tirgu Mures, Romania \\ 3. Department of Internal Medicine M3, University of Medicine and Pharmacy, Department of Cardiology, Institute for Cardiovascular Disease and Heart \\ Transplant, Tirgu Mures, Romania \\ 4. Department of Physiology, University of Medicine and Pharmacy, Tirgu Mures, Romania \\ 5. Department of Morphologic Sciences M1, University of Medicine and Pharmacy, Tirgu Mures, Romania
}

Objective: This study investigated predictors of progression of coronary atherosclerosis after percutaneous coronary intervention. Their identification may be useful in clinical practice. Methods: We retrospectively reviewed the database of the Cardiology Department of the Cardiovascular Disease and Heart Transplant Institute in Tirgu Mures from January 2012 to December 2015 and identified 180 patients readmitted after successful percutaneous coronary intervention. The t-test, chi-square test, Fisher's exact test, and mono- and multivariate analyses were used to evaluate associations between the patients' clinical and angiographic characteristics and the progression of coronary atherosclerosis. Results: The pre-percutaneous coronary intervention atherosclerotic burden was associated with a higher number of new coronary lesions at readmission. Hypertension and the placement of more than one bare-metal stent in the right coronary artery were associated with increased odds of the progression of coronary atherosclerosis. The use of drug-eluting stents at the index percutaneous coronary intervention and a greater number of drug-eluting stents in the left anterior descending artery were associated with a decreased chance of the progression of coronary atherosclerosis. Conclusions: A massive atherosclerotic load at index percutaneous coronary intervention and hypertension were predictors of the progression of coronary artery atherosclerosis. The number, type, and localisation of the stent at the index percutaneous intervention could influence the progression of coronary atherosclerosis. Further research is needed to identify other potential predictors and to determine how to optimize the treatment of known predictors.

Keywords: progression of coronary atherosclerosis, percutaneous coronary intervention, drug-eluting stents, bare-metal stent

Received 23 July 2018 / Accepted 28 August 2018

\section{Introduction}

The impressive progress in coronary stents has been accompanied by a considerable decrease in the need for revascularisation related to the target vessel treated at baseline [1]. Preventing the progression of coronary atherosclerosis by involving new vascular territories after successful percutaneous coronary intervention (PCI) is considered an attractive target. The present study aimed to assess the factors associated with the progression of coronary atherosclerosis after PCI. Their identification and treatment could influence the post-PCI prognosis of patients.

\section{Methods}

In order to identify predictors of the progression of coronary atherosclerosis, we reviewed the files of patients hospitalised in the Cardiology Department of the Cardiovascular Disease and Heart Transplant Institute in Tirgu Mures after successful PCI from January 2012 to December 2015. Only patients with coronary angiography upon readmission were included in the study.

* Correspondence to: Violeta Dinesch

E-mail: doc_violeta@yahoo.com
The progression of coronary atherosclerosis was defined as follows:

- a reduction of $\geq 10 \%$ in the diameter of a pre-existing stenosis $\geq 50 \%$

$-30 \%$ reduction in the diameter of a pre-existing stenosis $<50 \%$

- the progression of stenosis to occlusion

The risk factors for cardiovascular disease, comorbidities, details related to the stent used at index PCI (type, number, localisation), and medication after the index PCI were used to compare patients in the group who experienced the progression of coronary atherosclerosis (group B) to those in whom it did not occur (group A).

The data were analysed using the STATA program (version 14.0, Stata Corporation, College Station, TX, USA). Continuous variables were expressed as mean \pm standard deviation and were compared using the statistical significance t-tests and linear regression. The categorical variables were expressed as frequency and proportions. Comparisons were made using contingency tables, the chi-square test, and Fisher's exact test. In order to determine the meaning, significance, and strength of the relationships between the variables, logistic regression was used, with the result of 
the mono- and multivariate analyses being described as an odds ratio (OR) associated with a confidence interval of $95 \%$. A p value of 0.05 was considered statistically significant.

The study design was approved by the institutional ethics review board, and all patients provided informed consent.

\section{Results}

In 137 patients (76.11\%), at least one of the conditions for the progression of coronary atherosclerosis was met, while lesion regression was not found in any patients. Forty-three patients (23.88\%) showed stable coronary lesions.

The massive atherosclerotic load found on the index PCI was associated with a higher number of new post-PCI lesions at readmission $(\mathrm{p}=0.0002$, rho $=0.278)$, suggesting the progression of coronary lesions (Table I).

The mean duration of follow-up was $29 \pm 32$ months for group A and $31 \pm 32$ months for group B (OR 1.001, $\mathrm{p}=0.958)$. The progression of coronary atherosclerosis occurred in the coronary stents in 32 patients $(23.35 \%)$, in the native coronary arteries in 64 patients $(46.71 \%)$, and both in the stents and in the native coronary arteries in 41 patients (29.92\%).

The demographic and clinical characteristics of the patients are shown in Table II.

A lack of anginal symptoms was a good predictor for group A (OR 0.36, p = 0.018), while acute coronary syn-

Table I. Results of the pre- $\mathrm{PCl}$ and readmission coronarography

\begin{tabular}{llcc}
\hline \multicolumn{2}{c}{ Parameter } & \multicolumn{1}{c}{$\begin{array}{c}\text { Pre-PCI } \\
\text { coronarography }\end{array}$} & $\begin{array}{c}\text { Readmission } \\
\text { coronaro- } \\
\text { graphy }\end{array}$ \\
\hline \multirow{4}{*}{$\begin{array}{l}\text { Number of } \\
\text { coronary lesions }\end{array}$} & Monovascular & $78(43.33)$ & $72(40)$ \\
\cline { 2 - 4 } & Bivascular & $63(35)$ & $27(15)$ \\
\cline { 2 - 4 } & Trivascular & $34(18.89)$ & $10(5.56)$ \\
\cline { 2 - 4 } & $>3$ & $5(2.78)$ & 0 \\
\cline { 2 - 4 } Coronary lesion & Without lesions & 0 & $71(39.44)$ \\
\cline { 2 - 4 } localisation & $\begin{array}{l}\text { Left main } \\
\text { coronary artery }\end{array}$ & $6(1.8)$ & $4(2.22)$ \\
\cline { 2 - 4 } & LAD & $128(39.38)$ & $43(23.88)$ \\
\cline { 2 - 4 } & $\begin{array}{l}\text { RCA } \\
\text { coft circumflex }\end{array}$ & $60(18.46)$ & $18(10)$ \\
\cline { 2 - 4 } & $\begin{array}{l}\text { Other coronary } \\
\text { artery }\end{array}$ & $43(14.15)$ & $43(23.88)$ \\
\hline
\end{tabular}

Data are expressed as number (\%).

LAD: Left anterior descending artery; PCl: percutaneous coronary intervention; RCA: right coronary artery

Table II. Demographic and clinical characteristics: group A vs group B

\begin{tabular}{|c|c|c|c|c|}
\hline & Parameter & $\begin{array}{c}\text { Group A } \\
43(23.88)\end{array}$ & $\begin{array}{c}\text { Group B } \\
137(76.11)\end{array}$ & $\mathbf{p}$ \\
\hline Age, years & & $61.17 \pm 8.34$ & $62.25 \pm 10.38$ & $0.595^{\star}$ \\
\hline Male sex & & $32(74.41)$ & $99(72.26)$ & $0.745^{\star \star}$ \\
\hline \multirow{4}{*}{ Cardiovascular risk factors } & Hypertension & $34(79.06)$ & $126(91.19)$ & $0.004^{\star *}$ \\
\hline & Diabetes mellitus & $7(16.27)$ & $30(21.89)$ & $0.428^{\star *}$ \\
\hline & Obesity & $15(34.83)$ & $35(25.54)$ & $0.225^{\star \star}$ \\
\hline & Smoking & $4(9.3)$ & 19 (13.86) & $0.738^{\star *}$ \\
\hline \multirow{4}{*}{ Comorbidities } & Prior myocardial infarction & $18(41.86)$ & $72(52.55)$ & $0.216^{\star \star}$ \\
\hline & $\begin{array}{l}\text { Prior aortocoronary } \\
\text { bypass }\end{array}$ & $2(4.65)$ & $7(5.1)$ & $1^{* *}$ \\
\hline & eGFR $\leq 60 \mathrm{ml} / \mathrm{min} / 1.73 \mathrm{~m}^{2}$ & $8(18.6)$ & $35(25.54)$ & $0.352^{\star \star}$ \\
\hline & Ejection fraction $<50 \%$ & $8(18.6)$ & $32(23.35)$ & $0.516^{\star \star}$ \\
\hline \multirow{2}{*}{ Type of readmission } & Chronic & $37(86.36)$ & $86(67.15)$ & \multirow{2}{*}{$0.016^{\star \star}$} \\
\hline & Emergency & $6(9.3)$ & $45(32.8)$ & \\
\hline \multirow{3}{*}{ Diagnostic at readmission } & Unstable angina & $3(6.97)$ & $33(24.08)$ & $0.015^{\star \star \star}$ \\
\hline & Acute myocardial infarction & 0 & $9(6.56)$ & $0.117^{\star \star \star}$ \\
\hline & Cardiological reassessment & $12(27.9)$ & $17(12.4)$ & $0.015^{\star \star \star}$ \\
\hline Number of stents/patient & & $1.62 \pm 0.92$ & $1.54 \pm 0.96$ & $0.313^{\star \star \star}$ \\
\hline \multirow{2}{*}{ Type of stent } & DES & $18(41.86)$ & $35(25.54)$ & $0.038^{\star \star}$ \\
\hline & BMS & $28(65.11)$ & $107(78.1)$ & $0.069^{\star *}$ \\
\hline \multirow{5}{*}{$\begin{array}{l}\text { DES localisation } \\
\text { at index } \mathrm{PCl}\end{array}$} & Left main coronary artery & $1(2.32)$ & $3(2.18)$ & $1^{\star \star \star}$ \\
\hline & LAD & $11(25.58)$ & $22(16.05)$ & $0.154^{\star \star \star}$ \\
\hline & RCA & $6(13.95)$ & $11(8.02)$ & $0.243^{\star \star \star}$ \\
\hline & Left circumflex coronary artery & $3(6.97)$ & $6(4.37)$ & $0.446^{\star \star \star}$ \\
\hline & Other coronary artery & $3(6.97)$ & $5(3.64)$ & $0.398^{\star \star \star}$ \\
\hline \multirow{4}{*}{$\begin{array}{l}\text { BMS localisation } \\
\text { at index } \mathrm{PCl}\end{array}$} & Left main coronary artery & $1(2.32)$ & $1(0.72)$ & $0.421^{\star \star \star}$ \\
\hline & LAD & $19(44.18)$ & $56(40.87)$ & $0.686^{\star \star \star}$ \\
\hline & RCA & $4(9.3)$ & $40(29.19)$ & $0.007^{\star \star \star}$ \\
\hline & Left circumflex coronary artery & $8(18.6)$ & $22(16.05)$ & $0.689^{\star \star}$ \\
\hline \multirow{4}{*}{ Medical therapy } & Aspirin & $16(37.2)$ & $57(41.6)$ & $0.612^{\star \star}$ \\
\hline & Dual platelet anti-aggregation & $24(55.81)$ & $68(49.6)$ & $0.458^{\star *}$ \\
\hline & Angiotensin-converting enzyme inhibitor & $21(48.83)$ & $90(66.35)$ & $0.041^{\star *}$ \\
\hline & Statin & $34(79.06)$ & $94(68.61)$ & $0.153^{\star \star}$ \\
\hline
\end{tabular}


drome statistically correlated with group B (OR 5.98, p = 0.004). Stable angina symptomatology did not indicate a significant association with either of the groups (OR 0.7, $\mathrm{p}=0.341)$.

The use of drug-eluting stents at the index PCI and a greater number of drug-eluting stents at the left anterior descending artery (LAD) level decreased the chances for the patient to have progression of coronary atherosclerosis (OR 0.466, p = 0.04, and OR 0.52, p = 0.05) (Table III).

The use of bare-metal stents at the index PCI doubled the chances of the patient being in group B (OR 2), but the statistical significance was poor $(\mathrm{p}=0.072)$. Instead, the placement of more than one bare-metal stent in the right coronary artery almost tripled the chances of being in group B (OR 2.81, $\mathrm{p}=0.022$ ) (Table III).

The progression of coronary atherosclerosis in the native coronary artery occurred most frequently in the LAD and the right coronary artery (OR 20.66, $\mathrm{p}=0.004$ and $\mathrm{OR}$ $10.52, p=0.002$ ) (Figure 1). Of the cardiovascular risk factors, only hypertension was statistically associated with the progression of atherosclerotic lesions $(p=0.004)$. We found a statistically significant association between the use of angiotensin-converting-enzyme inhibitors (ACEI) and the progression of coronary atherosclerosis $(\mathrm{p}=0.041)$.

\section{Discussion}

In our study population, the predictors for the progression of coronary atherosclerosis after successful PCI were a greater pre-PCI atherosclerotic burden, in particular hypertension, and the placement of more than one baremetal stent in the right coronary artery. The use of drugeluting stents and a greater number of drug-eluting stents in the LAD were associated with a lack of progression of coronary atherosclerosis. In the native coronary artery, progression of atherosclerosis occurred more frequently in the $\mathrm{LAD}$ and in the right coronary artery territory.

Pre-PCI multi-vascular coronary lesions are commonly associated with the development of new post-PCI coronary lesions [2,3]. In agreement with previous research, a positive correlation was found in our study between the massive atherosclerotic load found on pre-PCI coronarography and multi-vascular coronary heart disease upon readmission, suggesting the progression of existing coronary lesions or the occurrence of new lesions. The progression of coronary atherosclerosis was found in two-thirds of our patients, but regression was not found in any patients.

Hypertension is a well-known atherogenic risk factor [4]. In our study, hypertension was the only cardiovascular risk factor associated with progression of coronary

Table III. Correlation between the progression of coronary atherosclerosis and the stents type, their number, and localisation in coronary artery

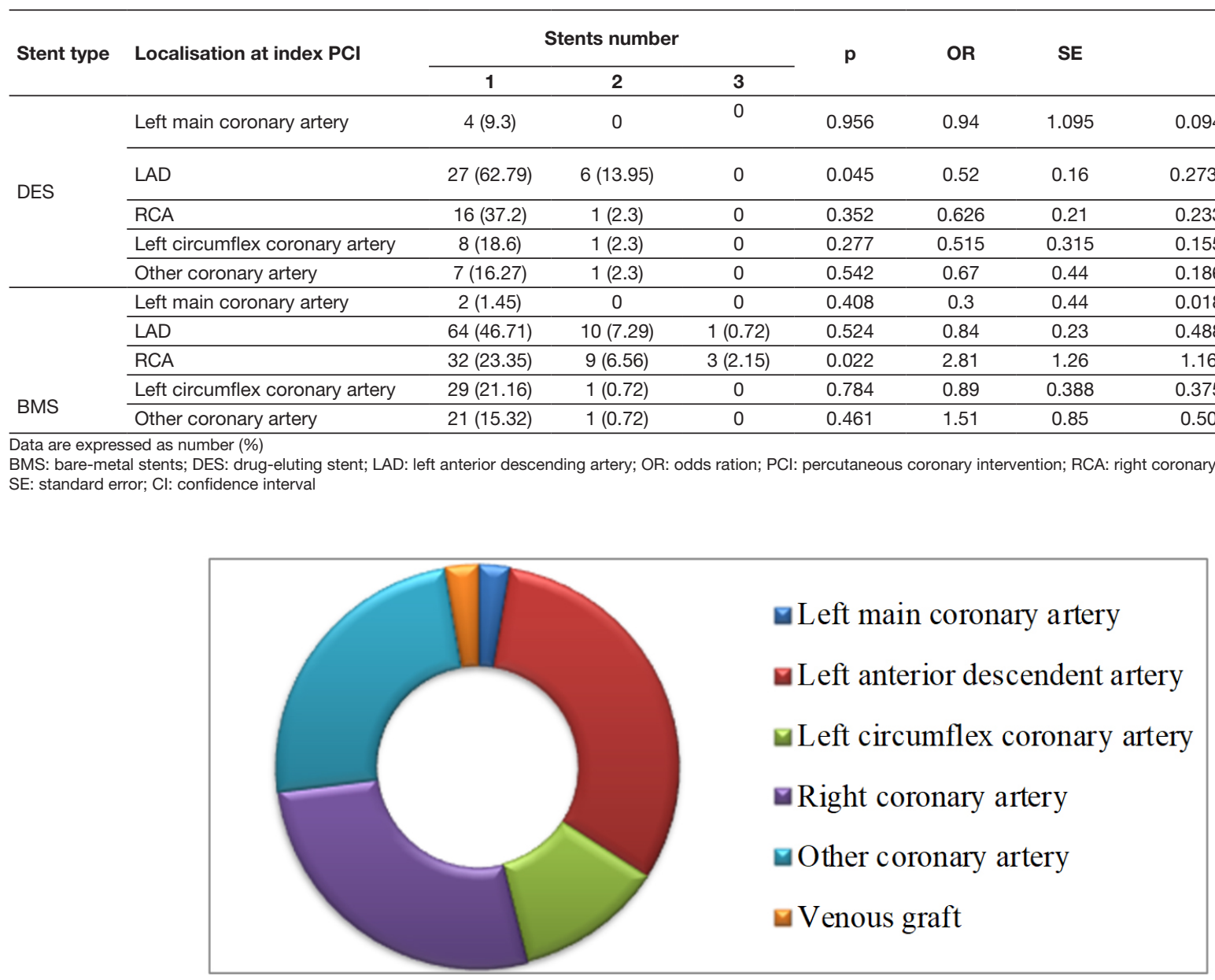

Fig. 1. Progression of atherosclerosis in the native coronary artery by location 
atherosclerosis. Similar to our study, Borges et al. found that hypertension, along with male sex, is a predictive factor for the progression of coronary atherosclerosis [5]. The importance of aggressive treatment of all risk factors for cardiovascular disease is highlighted in the COURAGE trial, where patients with optimal medical therapy (intensive pharmacologic therapy and lifestyle intervention) had fewer cardiovascular events than PCI-treated patients [6].

The superiority of using drug-eluting stents compared to bare-metal stents in order to reduce the need for revascularisation related to the initially treated target vessel has been demonstrated in several trials $[7,8]$. However, in a study involving 428 patients randomised to PCI with drug-eluting stents or bare-metal stents, Zelwegger et al. concluded that the progression of coronary atherosclerosis was similar between the two groups regardless of the type of stent used in the index PCI [9]. In our study, the use of drug-eluting stents at the index PCI was a predictor for the absence of the progression of coronary atherosclerosis.

The progression of coronary atherosclerosis in the native coronary arteries has shown conflicting data. In the CASS trial, in patients treated with $\mathrm{CABG}$, the progression of coronary atherosclerosis was more aggressive in LAD territory [10]. Additionally, Borges et al. found that PCI-treated patients had more progression of coronary atherosclerosis in LAD territory [5]. On the contrary, in the INTACT trial, the progression of coronary atherosclerosis occurred more frequently in the right coronary artery territory [11]. In our study, first the LAD and then the right coronary artery territory was associated with the progression of coronary atherosclerosis. Interestingly, in our study, a greater number of drug-eluting stents in the LAD was associated with a lack of progression of coronary atherosclerosis, while the placement of more than one bare-metal stent in the right coronary artery was a predictor for the progression of coronary atherosclerosis. We did not find any data in the literature about the association between placement, the number of drug-eluting stents/bare-metal stents at index PCI, and the progression of coronary atherosclerosis.

Numerous studies have demonstrated the beneficial effects of ACEI in patients with ischemic coronary artery disease, not only in reducing blood pressure but also in stabilising the atheromatous plaque and inducing the regression of uncalcified coronary stenoses $[12,13]$. In contrast, our study found a statistically significant association between the presence of ACEI and the progression of coronary atherosclerosis. This can be explained from at least from two points of view. First, hypertension, whose first line of treatment is ACEI, was statistically associated with the same group. Secondly, the study did not investigate the type and intensity of treatment with ACEI, and the effect of ACEI is not equal for all components of the class.

Our study has a few limitations. The present study was a single centre, retrospective study, and thus, our conclusions are not generalisable. Second, the small sample size reduced the statistical power to detect association with other predictive factors for the progression of coronary atherosclerosis.

\section{Conclusion}

A massive pre-PCI atherosclerotic load and hypertension were predictors of the progression of coronary artery atherosclerosis. The number, type, and localisation of the stent at the index percutaneous intervention could influence the progression of coronary atherosclerosis. Further research is needed in order to identify other potential predictors and to determine how to optimize the treatment of known predictors.

\section{Acknowledgements}

This research did not receive any specific grant from funding agencies in the public, commercial, or not-for-profit sectors.

\section{Conflict of interest}

None to declare.

\section{Author's contribution}

VD - Conceptualization, data curation, formal analysis, investigation, methodology, supervision, validation, visualization, writing original draft, writing review and editing MD - Conceptualization, data curation, formal analysis, investigation, supervision, writing review and editing IVS - Conceptualization, formal analysis, methodology, validation, writing review and editing

CM - Data curation, formal analysis, methodology, visualization, writing review and editing

BVH - Conceptualization, data curation, formal analysis, visualization, writing review and editing

MB - Conceptualization, formal analysis, methodology;, supervision, validation, visualization, writing review and editing

\section{References}

1. Bønaa K, Mannsverk J, Wiseth R, et al. - Drug-eluting or bare-metal stents for coronary artery disease. N Engl J Med. 2016;375:1242-1252.

2. Kaneko H, Yajima J, Oikawa $\mathrm{Y}$, et al. - Long-term incidence and prognostic factors of progression of new coronary lesions in Japanese coronary artery disease patients after percutaneous coronary intervention. Heart Vessels. 2014;29:437-442.

3. Park MW, Seung KB, Kim PJ, et al. - Long-term percutaneous coronary intervention rates and associated independent predictors for progression of nonintervened nonculprit coronary lesions. Am J Cardiol. 2009;104:648-652.

4. Sipahi I, Tuzcu EM, Schoenhagen P, et al. - Effects of normal, prehypertensive, and hypertensive blood pressure levels on progression of coronary atherosclerosis. J Am Coll Cardiol. 2006;48:833-838.

5. Borges JC, Lopes N, Soares PR, et al. - Five-year follow-up of angiographic disease progression after medicine, angioplasty, or surgery. J Cardiothorac Surg. 2010;5:91.

6. Boden WE, O'Rourke RA, Teo KK, et al. - Optimal medical therapy with or without $\mathrm{PCl}$ for stable coronary disease. $\mathrm{N}$ Engl $\mathrm{J}$ Med. 2007;356:1503-1516.

7. Ellis SG, Stone GW, Cox DA, et al. - Long-term safety and efficacy with paclitaxel-eluting stents: 5-year final results of the TAXUS IV clinical trial (TAXUS IV-SR: treatment of de novo coronary disease using a single paclitaxel-eluting stent). JACC Cardiovasc Interv. 2009;2:1248-1259.

8. Bangalore S, Kumar S, Fusaro M, et al. - Short- and long-term 
outcomes with drug-eluting and bare-metal coronary stents: a mixedtreatment comparison analysis of 117762 patient-years of follow-up from randomized trials. Circulation. 2012;125:2873-2891.

9. Zellweger MJ, Kaiser C, Jeger R, et al. - Coronary artery disease progression late after successful stent implantation. J Am Coll Cardiol. 2012;59:793-799.

10. Alderman EL, Corley SD, Fisher LD, et al. - Five-year angiographic follow-up of factors associated with progression of coronary artery disease in the coronary artery surgery study (CASS). CASS participating investigators and staff. J Am Coll Cardiol. 1993;22:1141-1154.

11. Lichtlen PR, Nikutta P, Jost $S$, et al. - Anatomical progression of coronary artery disease in humans as seen by prospective, repeated, quantitative coronary angiography. Relation to clinical events and risk factors. The INTACT Study Group. Circulation. 1992;86:828-838.

12. Fox KM, EURopean trial On reduction of cardiac events with Perindopril in stable Artery disease Investigators - Efficacy of perindopril in reduction of cardiovascular events among patients with stable coronary artery disease: randomised, double-blind, placebo-controlled, multicentre trial (the EUROPA study). Lancet. 2003;362:782-788.

13. Gude D - Angiotensin-converting enzyme inhibitors in lipid metabolism and atherosclerosis: An ace up the sleeve? J Sci Soc. 2014;41;59-60. 